\title{
Role of Nampt overexpression in a rat model of Hashimoto's thyroiditis and its mechanism of action
}

\author{
JIA-ZHEN TANG, WEN-QIONG XU, FU-JUAN WEI, YANG-ZHEN JIANG and XIAO-XUE ZHENG
}

\begin{abstract}
Department of Endocrinology, The First Affiliated Hospital of Nanchang University, Nanchang, Jiangxi 330006, P.R. China
\end{abstract}
Received April 30, 2019; Accepted December 3, 2019

DOI: $10.3892 /$ etm.2020.8539

\begin{abstract}
The present study was designed to investigate the role of nicotinamide phosphoribosyltransferase (Nampt) overexpression in a rat model of Hashimoto's thyroiditis (HT) and its mechanism of action. A rat model of HT was constructed, and the HT rats were injected with an adenoviral expression vector carrying the Nampt gene. The expression of Nampt and Toll-like receptor 4 (TLR4) in thyroid tissues was examined using immunohistochemistry (IHC), RT-qPCR and western blot analyses. Serum anti-thyroglobulin antibodies (TGAb) and anti-thyroid peroxidase antibodies (TPOAb) were measured using chemiluminescence method. Hematoxylin and eosin (H\&E) and IHC staining of the rat thyroid tissues showed destroyed thyroid follicles and monocyte infiltration, as well as increased Nampt expression in the thyroid tissues of rats with HT. Furthermore, it was found that Nampt overexpression led to increased severity of inflammatory infiltration in thyroid tissues and increased levels of TPOAb in the serum of HT rats; however, the serum TGAb level was not affected by Nampt overexpression. In addition, Nampt overexpression promoted TLR4 expression in HT rats. In conclusion, it was demonstrated that Nampt was strongly expressed in the capillary region of HT rats thyroid tissues. The Nampt mRNA level was increased but the Nampt protein level was decreased in the thyroid tissues of rats with HT. Nampt overexpression has a promotive effect on HT progression, and this effect was related to TLR4. This study suggests that inhibition of Nampt activity may be valuable in the treatment of HT.
\end{abstract}

\section{Introduction}

Hashimoto's thyroiditis (HT) is a chronic autoimmune thyroid disease characterized by autoimmune-mediated destruction of

Correspondence to: $\mathrm{Dr}$ Wen-Qiong Xu, Department of Endocrinology, The First Affiliated Hospital of Nanchang University, 17 Yong Wai Zheng Street, East Lake, Nanchang, Jiangxi 330006, P.R. China

E-mail: xuwenqiongaa@126.com

Key words: Hashimoto's thyroiditis, nicotinamide phosphoribosyltransferase, Nampt, Toll-like receptor 4, anti-thyroid peroxidase antibodies, anti-thyroglobulin antibodies the thyroid gland (1). Individuals with HT may have no symptoms early on. However, over time, the thyroid may enlarge, forming a painless goiter, and most patients eventually develop hypothyroidism $(2,3)$. The diagnosis of HT relies on the demonstration of circulating antibodies to thyroid peroxidase and thyroglobulin (4). Despite the large number of studies, HT remains a complex and expanding disease that awaits further understanding and novel forms of treatment.

Nicotinamide phosphoribosyltransferase (Nampt), also known as pre-B cell colony-enhancing factor (PBEF) or visfatin, is a member of the nicotinic acid phosphoribosyltransferase family (5). It is the rate limiting component in the mammalian NAD biosynthesis pathway. In addition, Nampt acts as a cytokine with immunomodulating properties and an adipokine with anti-diabetic properties (6-8). Recently, more and more studies have revealed the association between Nampt and autoimmune thyroid disease (AITD). Nampt is overexpressed in leukocytes and thyroid tissues of patients with Graves' disease $(4,9,10)$. The serum concentration of Nampt was also found to be increased in Graves' disease (11). Clinical studies and animal experiments have shown elevated plasma Nampt concentrations in hyperthyroidism and hypothyroidism groups $(12,13)$. Li and $\mathrm{Li}$ reported that plasma Nampt levels were higher in patients with HT than that noted in controls (14). All of these studies revealed the changes in Nampt expression in AITD; however, studies on the exact role and the mechanisms of Nampt in AITD are rare.

Herein, a rat model of HT was constructed and it was ascertained whether Nampt overexpression contributes to the development of HT. Furthermore, the effect of Nampt overexpression on Toll-like receptor 4 (TLR4) in HT was also investigated.

\section{Materials and methods}

Animal experiments. All the animal procedures were approved by the Ethics Committee of The First Affiliated Hospital of Nanchang University (Nanchang, Jiangxi, China). A total of 38 female SD rats (8 weeks old) were obtained from Hunan Slac Jingda Laboratory Animal Co., Ltd. (Changsha, Hunan, China). The rats were randomly assigned into two groups: the control group (Control group, $\mathrm{n}=8$ ) and the Hashimoto's thyroiditis group (Model group, $n=9$ ). Rats in the model group were injected with $0.2 \mathrm{ml}$ porcine thyroglobulin $(4 \mathrm{mg} / \mathrm{ml}$; Huayang Zhenglong Biochemical Products) emulsified in complete 
Freund's adjuvant (CFA; MP Biomedicals) at the footpad, and with a booster injection of an equal dose of porcine thyroglobulin in incomplete Freund's adjuvant (IFA) performed 2 and 4 weeks later. Rats in the control group were treated with phosphate-buffered saline (PBS) instead of porcine thyroglobulin at the same time. The rats in the normal and the model groups were maintained under standard laboratory conditions, and allowed access to deionized water or $0.05 \%$ $\mathrm{Na}$ I solution (Shanghai Zhanyun Chemical Co., Ltd.). One week after the third immunization, the thyroid tissues were collected for testing. Empty adenoviral vector and adenoviral expression vector carrying the Nampt gene were provided by Abmgoodchina Inc. Rats with HT were injected with $10^{9} \mathrm{vp}$ of empty adenoviral vector (Model+NC group, $n=9$ ) or adenoviral expression vector carrying the Nampt gene (Model+Nampt group, $n=9$ ) through tail vein for 3 days. One week later, thyroid tissues and serum samples were collected for testing.

Hematoxylin and eosin (H\&E) staining. Thyroid tissues were fixed in formalin, and then embedded in paraffin. After deparaffinization and rehydration, the sections were stained with hematoxylin solution for $3 \mathrm{~min}$ followed by differentiation in acid ethanol for $15 \mathrm{sec}$. Following rinsing in distilled water, the sections were then stained with eosin solution (Boster) for 3 min, dehydrated with graded alcohol, and cleared in xylene. The slides were observed under an Olympus CX41 microscope (magnification, x200; Olympus, Tokyo, Japan).

Immunohistochemistry (IHC). Sections were baked at $65^{\circ} \mathrm{C}$ for $2 \mathrm{~h}$, followed by incubation with xylene for $20 \mathrm{~min}$ and graded ethanol for $25 \mathrm{~min}$. High temperature and high pressure citrate buffer was used to retrieve antigen. Endogenous peroxidase activity was quenched by incubation with $3 \% \mathrm{H}_{2} \mathrm{O}_{2}$ at room temperature for $10 \mathrm{~min}$. BSA (5\%) was added to the sections to block nonspecific staining. Antibodies against Nampt (cat. no. DF6059; Affinity Biosciences) and TLR4 (cat. no. bs-20594R; Beijing Biosynthesis Biotechnology Co., Ltd.) were diluted at 1:200, and the sections were incubated with the primary antibodies at $4^{\circ} \mathrm{C}$ overnight. The secondary antibody (cat. no. ZB-2301; ZSBIO) was used at a 1:100 dilution. The sections were incubated with the secondary antibody at $37^{\circ} \mathrm{C}$ for $30 \mathrm{~min}$. After washing with PBS, the sections were incubated with the DAB solution (CWBIO) and then counterstained with hematoxylin (Boster).

Reverse transcriptionquantitative polymerase chain reaction $(R T-q P C R)$. Total RNA was extracted using TRIzol reagent (CWBIO) and purified with an Ultrapure RNA Kit (CWBIO). The following primers were used in the present study: Nampt, 5'-ATGCCGTGAAAAGAAGACAG-3' (forward) and 5'-TCC AGTTGGTGAGCCAGTAG-3' (reverse); TLR4, 5'-AAGAGT CTAGCCGTCTTCAATC-3' (forward) and 5'-CAGCCAGCA ATAAGTATCAGG-3' (reverse); GAPDH, 5'-TACCCACGG CAAGTTCAA-3' (forward) and 5-ACCAGCATCACCCCA TTT-3' (reverse). All primers were synthesized by Sangon Biotech Co., Ltd. Total RNA was reverse transcribed into cDNA using a HiFiScript cDNA Synthesis Kit (CWBIO) in accordance with the manufacturer's instructions. qPCR was performed using UltraSYBR Mixture (CWBIO) with an initial hold step $\left(95^{\circ} \mathrm{C}\right.$ for $\left.10 \mathrm{~min}\right)$ and 40 cycles at $95^{\circ} \mathrm{C}$ for
$10 \mathrm{sec}, 57^{\circ} \mathrm{C}$ for $30 \mathrm{sec}$ and $72^{\circ} \mathrm{C}$ for $30 \mathrm{sec}$. Relative mRNA expression was analyzed using the $2^{(-\Delta \Delta \mathrm{Cq})}$ method (15).

Western blot analysis. Tissue samples were homogenized using liquid nitrogen and lysed in RIPA lysis buffer (Applygen Technologies Inc.) for $30 \mathrm{~min}$. Lysates were then centrifuged at $4^{\circ} \mathrm{C}$ for $10 \mathrm{~min}$ at $12,000 \mathrm{x}$ g, and the supernatants were collected. Total proteins were separated by SDS-polyacrylamide gel electrophoresis, and transferred onto PVDF membranes (Millipore). The membranes were incubated with the appropriate primary antibodies, including rabbit polyclonal anti-Nampt (cat. no. DF6059; Affinity Biosciences; dilution, 1:500), rabbit polyclonal anti-TLR4 (cat. no. bs-20594R; Beijing Biosynthesis Biotechnology Co., Ltd.; dilution, 1:1,000) and mouse monoclonal anti-GAPDH (cat. no. TA-08; ZSBIO; dilution, $1: 2,000)$ at $4{ }^{\circ} \mathrm{C}$ overnight, followed by incubation with HRP-conjugated goat anti-rabbit IgG $(\mathrm{H}+\mathrm{L})$ (cat. no. ZB-2301; ZSBIO; dilution, 1:2,000) or HRP-conjugated goat anti-mouse $\operatorname{IgG}(\mathrm{H}+\mathrm{L})$ (cat. no. ZB-2305; ZSBIO; dilution, $1: 2,000)$ at $4{ }^{\circ} \mathrm{C}$ for $2 \mathrm{~h}$. Signals were visualized with the SuperSignal ${ }^{\circledR}$ West Pico Chemiluminescent Substrate (Thermo Fisher Scientific, Inc.), and the band density was quantified using Quantity One software (version 4.6.9; Bio-Rad Laboratories, Inc.).

Measurement of anti-thyroglobulin and anti-thyroid peroxidase antibodies. The thyroglobulin protein and thyroid peroxidase were purchased from Beijing Biosynthesis Biotechnology Co., Ltd. Anti-thyroglobulin antibodies (TGAb) and anti-thyroid peroxidase antibodies (TPOAb) in serum were assessed by enzyme-linked immunosorbent assay on an automatic chemiluminescence immunoassay instrument (ADVIA Centaur CP, Siemens Medical Solutions Diagnostics), in accordance with the manufacturer's guidelines.

Statistical analysis. Statistical analysis was performed using SPSS 19.0 software (IBM Corp.), and the data are presented as means \pm SD. Comparisons of data between groups were made using one-way analysis of variance (ANOVA) followed by Bonferroni's post hoc test. $\mathrm{P}<0.05$ was considered to indicate a statistically significant difference.

\section{Results}

Construction of an HT model in rats. H\&E staining of thyroid tissues are shown in Fig. 1. Rats in the control group (Fig. 1A) displayed intact and even-distributed thyroid follicles, and lymphocyte infiltration was barely presented in the thyroid tissues. The pathological grading of the sections in the model group was grade I. It was shown that the thyroid follicles in the HT rats were disordered and destroyed, and lymphocyte infiltration was observed around the thyroid follicles (Fig. 1B-D).

Expression of Nampt in the thyroid tissues of the HT rats. The rats were injected with porcine thyroglobulin to induce HT model, and IHC was performed to detect Nampt protein in the thyroid tissues of HT rats. As shown in Fig. 2, Nampt was widely expressed in thyroid follicles and blood cells. The immunological staining of inflammatory infiltrating cells was obviously stronger in the model group than that noted in the control group. 

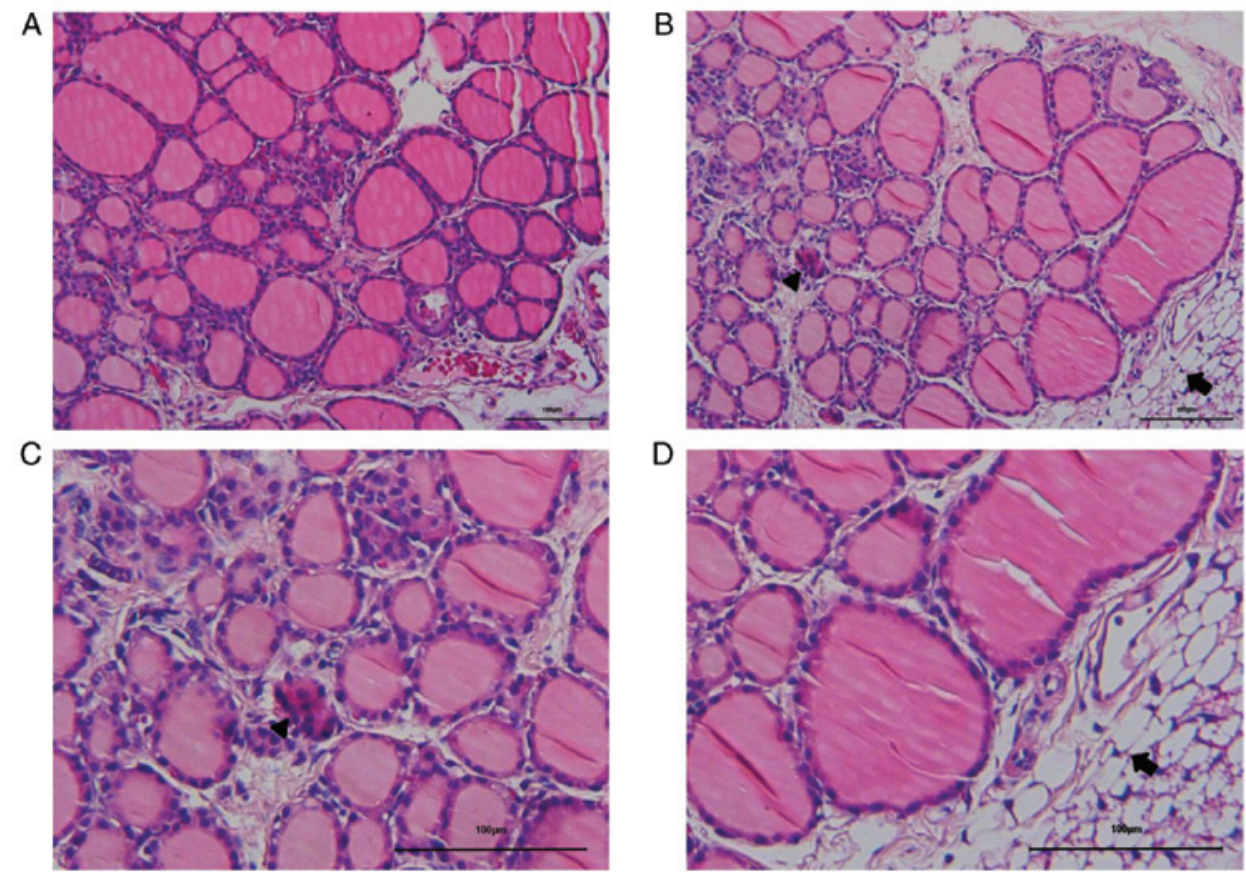

Figure 1. Hematoxylin and eosin staining of rat thyroid tissues. (A) Control group (magnification, x200). (B) Model group (magnification, x200). (C) High magnification of an area in (B) showing inflammatory cells infiltration. (D) High magnification of an area in (B) showing follicle cell breakdown. Arrow, follicle cell breakdown; arrowhead, inflammatory cell infiltration; scale bar, $100 \mu \mathrm{m}$.

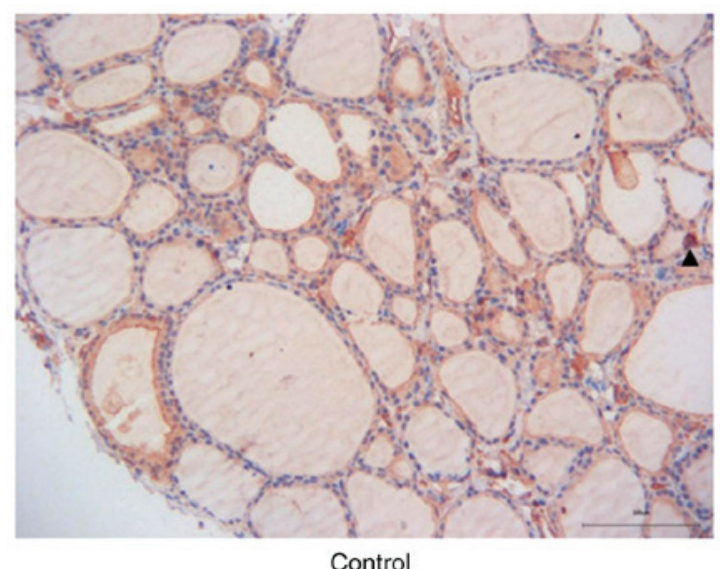

Control

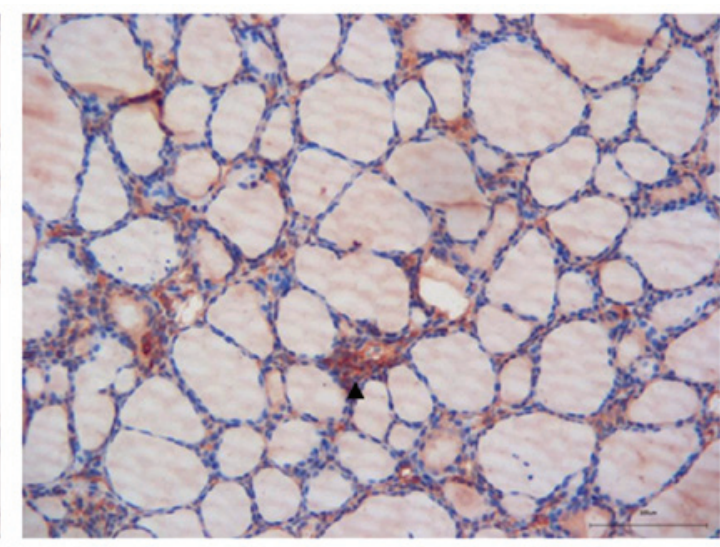

Model

Figure 2. Immunohistochemical staining of Nampt in rat thyroid tissues (magnification, x200) in the Control and Model groups. Arrowhead, Nampt-positive. Nampt, nicotinamide phosphoribosyltransferase.

Effect of Nampt on TLR4 expression in HT rats. We examined Nampt mRNA and protein levels in the thyroid tissues using RT-qPCR and western blot analyses. The Nampt mRNA level was significantly increased in rats with HT compared with the control rats; however, the Nampt protein level was decreased in the model group. Compared with the HT rats injected with empty adenoviral vector, we found Nampt expression was significantly increased both at the mRNA level and the protein level in the HT rats injected with adenoviral expression vector carrying the Nampt gene (Fig. 3A and B).

To evaluate the effect of Nampt on TLR4 expression, the sections of rat thyroid tissues were stained with an antibody against TLR4. It was shown that the thyroid tissues had more extensive follicular atrophy and collapse, as well as more serious inflammatory infiltration in the model+Nampt group than in the model+NC group. TLR4 was mainly expressed in monocytes/macrophages and endothelial cells. We found that TLR4 was more strongly positive in the model+Nampt group than in the model+NC group (Fig. 3C). In addition, we examined TLR4 mRNA and protein levels in the thyroid tissues. The results revealed that TLR 4 mRNA and protein levels were significantly upregulated in the rats with HT compared with the control rats. Nampt overexpression resulted in increased TLR4 mRNA and protein levels in rats with HT (Fig. 3A and B) compared with the model+NC group.

Effect of Nampt on TGAb and TPOAb in HT rats. We detected serum concentrations of TGAb and TPOAb using chemiluminescence method. As shown in Fig. 4, the TGAb level was significantly higher in the model group than in 

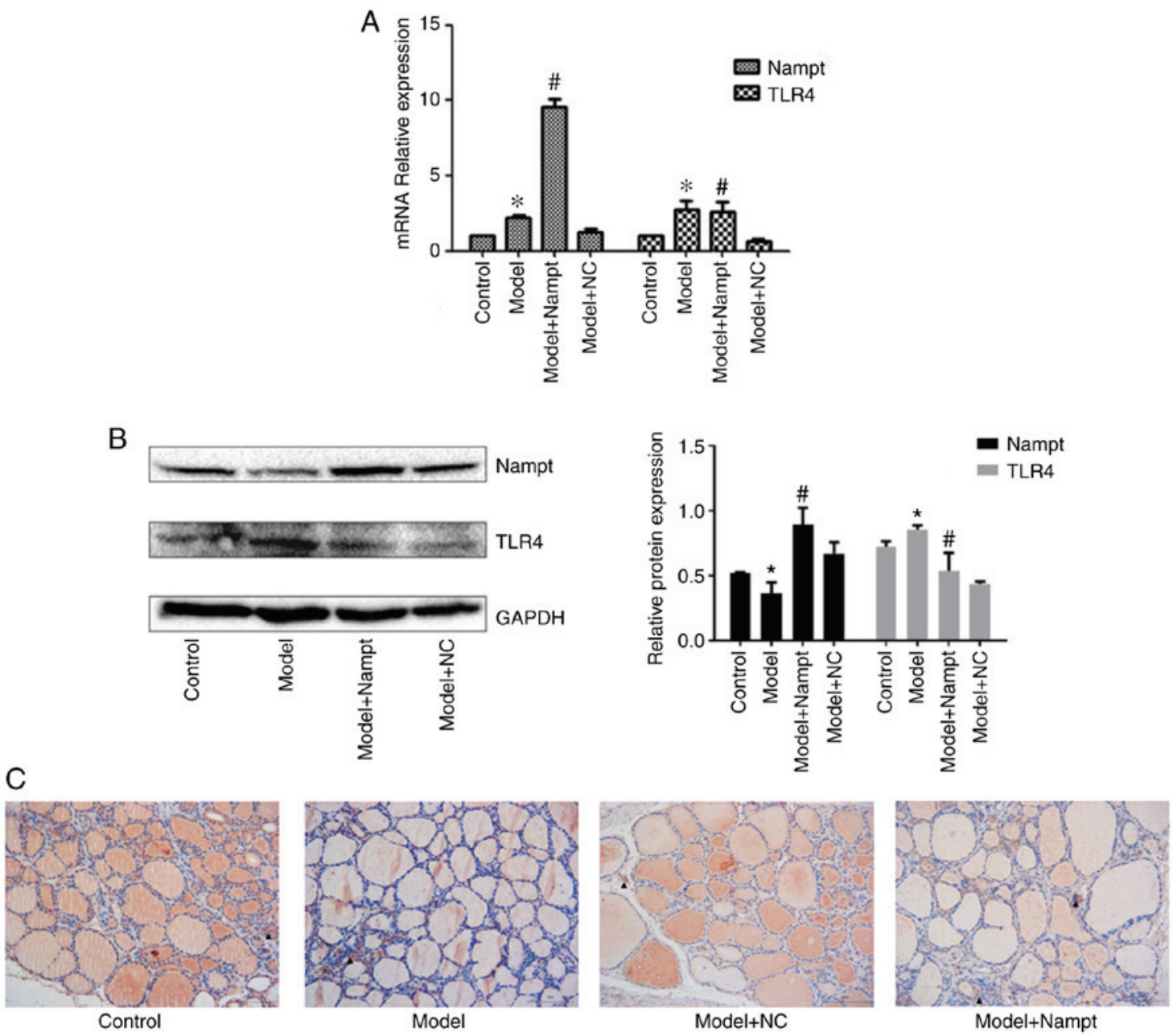

Figure 3. Effect of Nampt on TLR4 expression in HT rats. (A) Expression of Nampt and TLR4 mRNA in rat thyroid tissues. (B) Expression of Nampt and TLR4 protein in rat thyroid tissues. ${ }^{\mathrm{P}}<0.05$ compared with the control group; ${ }^{*} \mathrm{P}<0.01$ compared with the model+NC group. (C) Immunohistochemical staining of TLR4 in rat thyroid tissues (magnification, x200). Arrowhead, TLR4-positive. HT, Hashimoto's thyroiditis; Nampt, nicotinamide phosphoribosyltransferase; TLR4, Toll-like receptor 4.

the control group, while no difference in TPOAb level was detected between the two groups. Moreover, we found there was a significant increase in TPOAb level in the model+Nampt group compared with that in the model+NC group; however, we did not observe a change in TGAb level between the two groups.

\section{Discussion}

Nampt is involved in many important biological processes, including survival, apoptosis, metabolism and angiogenesis (16). As a cytokine, an elevated concentration of Nampt has been observed in several inflammatory and autoimmune disorders, such as sepsis, atherosclerosis, systemic lupus erythematosus and Crohn's disease (17-20). Previous studies have demonstrated an elevated plasma Nampt concentration in Hashimoto's thyroiditis (HT) patients (14). Nampt is present in plasma to behave both as a cytokine and an adipokine. The blood brings Nampt to all tissues of the body. In the present study, adenoviral expression vector carrying the Nampt gene was injected into rats through the tail vein to elevate the Nampt level in plasma. Elevated Nampt was transported to the thyroid gland by the blood to exert its local effects. The administration

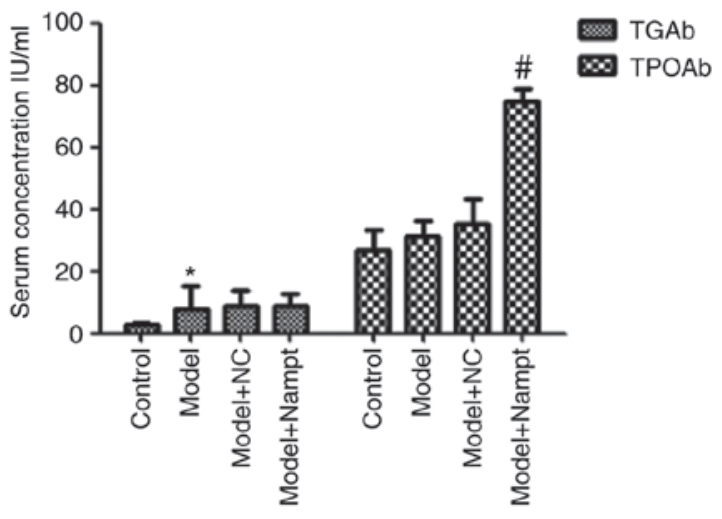

Figure 4. Effect of Nampt on serum concentration of TGAb and TPOAb in HT rats. ${ }^{*} \mathrm{P}<0.05$ compared with the control group; ${ }^{\prime} \mathrm{P}<0.01$ compared with the model+NC group. HT, Hashimoto's thyroiditis; Nampt, nicotinamide phosphoribosyltransferase; TGAb, anti-thyroglobulin antibodies; TPOAb, anti-thyroid peroxidase antibodies.

method used in this study could mimic the effect of Nampt on HT in human bodies. In this study, we found that Nampt was strongly expressed in the capillary region of HT rat thyroid tissues. The Nampt mRNA level was increased but the Nampt 
protein level was decreased in the thyroid tissues of rats with HT. Nampt has two different forms: intranuclear and secreted Nampt. In the thyroid tissues of the model group, the staining of Nampt in the capillary region of thyroid tissues was stronger in the model group than in the control group, suggesting that Nampt is more likely to enter the blood in a secreted form. The elevated secreted Nampt may lead to the decreased Nampt protein level in the thyroid tissues. Our hypothesis was consistent with a previous study reporting that plasma Nampt concentration was elevated in HT patients (14).

Autoimmune thyroid diseases are characterized by antibodies against thyroglobuline and thyroid peroxidase. Positive serum TPOAb and TGAb are found in the majority of HT patients. In the present study, an HT animal model was established by injecting porcine thyroglobulin. We found inflammatory infiltration in the thyroid tissues of the HT rats; however, only TGAb was significantly increased in the model group, suggesting that there were some differences between the animal model and HT subjects. This may be due to the fact that the thyroid injury was mild in the model group, and the disease duration of the model group was short. Furthermore, Nampt overexpression was demonstrated to lead to increased severity of inflammatory infiltration in thyroid tissues as well as increased TPOAb levels in the serum of HT rats; however, the serum TGAb level was not affected by Nampt overexpression. TPOAb is a diagnostic marker of HT, and TPOAb could cause thyroid cell damage through antibody-complement system-mediated $\mathrm{T}$ cell activation (21-23). Nampt overexpression led to the increased serum TPOAb level in HT rats, indicating that Nampt promotes HT progression. However, TGAb did not increase after Nampt overexpression, suggesting that thyroid follicle cells may not be attacked by Nampt overexpression.

Toll-like receptor 4 (TLR4) is a member of the TLR family which plays a key role in the innate immune system. TLR4 triggers inflammatory response, leading to $\mathrm{NF}-\kappa \mathrm{B}(\mathrm{NF}-\kappa \mathrm{B})$ activation and cytokine secretion (24-26). In periodontal ligament cells, there was a positive correlation between TLR2/4 and Nampt expression (27). A study by Camp et al reported that TLR4 is a receptor for Nampt; Nampt induces lung NF- $\kappa$ B transcriptional activities and inflammatory injury via direct ligation of TLR4 (28). In the present study, compared with the control rats, the mRNA and protein expression levels of TLR4 were significantly increased in rats with HT. Nampt overexpression promoted TLR4 expression in HT rats. It has been reported that TLR4 protein expression is regulated by many other factors in the inflammatory process, such as HMGB1, hyaluronan fragments, HSP60 and fibrinogen (29-32). This study demonstrated that TLR4 expression was also regulated by Nampt.

In the present study, the rats in the Model+NC group were injected with empty adenoviral vector. It has been reported that bioengineered adenovirus can stimulate the immune system (33), causing an in vivo immune response in rats. Therefore, a significant difference between the Model group and Model+NC group may exist. Interestingly, we found the difference between the Model+NC and Model groups was even more significant than that between the Model+Nampt and Model groups. This result indicated that intravenous injection of adenovirus inhibited the autoimmune response to the thyroid gland. Adenovirus particles act as antigens to induce humoral immune response. Humoral immune response competes with the autoimmune response to the thyroid gland, leading to a decrease in autoimmunity.

A previous study reported that the Nampt inhibitor FK866 modulated T cell-mediated immune responses and thereby was beneficial in immune-mediated disorders (34). We expected an inhibitory effect of Nampt silencing on thyroid inflammation and HT progression. Our further studies may focus on the effect of FK866 on HT in animal models.

A limitation of this study was that there was a nonspecific staining of colloid of thyroid follicles, and the staining influenced the evaluation of Nampt and TLR4 expression. Therefore, we did not evaluate the relative expression of Nampt and TLR4 in the IHC study.

In conclusion, this study revealed that Nampt was strongly expressed in the capillary region of HT rat thyroid tissues. The Nampt mRNA level was increased but the Nampt protein level was decreased in the thyroid tissues of rats with HT. Nampt overexpression had a promotive effect on HT progression, and this effect was related to TLR4. These results suggest that inhibition of Nampt activity may be valuable in the treatment of HT.

\section{Acknowledgements}

Not applicable.

\section{Funding}

This study was supported by the Natural Science Foundation of Jiangxi Province (grant no. 20171BAB205038) and the Jiangxi Provincial Department of Education (grant no. 150256).

\section{Availability of data and materials}

The datasets used and/or analyzed during the current study are available from the corresponding author on reasonable request.

\section{Authors' contributions}

JZT and WQX designed the study. JZT, FJW, YZJ and $\mathrm{XXZ}$ performed the experiments and collected the data. JZT, WQX and FJW conducted the statistical analysis and participated in data interpretation. JZT wrote the manuscript, and WQX revised the manuscript. All authors read and approved the manuscript and agree to be accountable for all aspects of the research in ensuring that the accuracy or integrity of any part of the work are appropriately investigated and resolved.

\section{Ethics approval and consent to participate}

All the animal procedures were approved by the Ethics Committee of The First Affiliated Hospital of Nanchang University (Nanchang, Jiangxi, China).

\section{Patient consent for publication}

Not applicable. 


\section{Competing interests}

The authors declare that they have no competing interests.

\section{References}

1. Caturegli P, De Remigis A and Rose NR: Hashimoto thyroiditis: Clinical and diagnostic criteria. Autoimmun Rev 13: 391-397, 2014.

2. Umar H, Muallima N, Adam JM and Sanusi H: Hashimoto's thyroiditis following Graves' disease. Acta Med Indones 42: $31-35,2010$

3. Ohye $\mathrm{H}$ and Sugawara M: Dual oxidase, hydrogen peroxide and thyroid diseases. Exp Biol Med (Maywood) 235: 424-433, 2010.

4. Sawicka-Gutaj N, Zybek-Kocik A, Klimowicz A, Kloska M, Mańkowska-Wierzbicka D, Sowiński J and Ruchała M: Circulating visfatin in hypothyroidism is associated with free thyroid hormones and antithyroperoxidase antibodies. Int J Endocrinol 2016: 7402469, 2016.

5. Dahl TB, Holm S, Aukrust P and Halvorsen B: Visfatin/NAMPT: A multifaceted molecule with diverse roles in physiology and pathophysiology. Annu Rev Nutr 32: 229-243, 2012.

6. Revollo JR, Grimm AA and Imai S: The regulation of nicotinamide adenine dinucleotide biosynthesis by Nampt/PBEF/visfatin in mammals. Curr Opin Gastroenterol 23: 164-170, 2007.

7. Choi SE, Fu T, Seok S, Kim DH, Yu E, Lee KW, Kang Y, Li X, Kemper B and Kemper JK: Elevated microRNA-34a in obesity reduces $\mathrm{NAD}^{+}$levels and SIRT1 activity by directly targeting NAMPT. Aging Cell 12: 1062-1072, 2013.

8. Garten A, Schuster S, Penke M, Gorski T, de Giorgis T and Kiess W: Physiological and pathophysiological roles of NAMPT and NAD metabolism. Nat Rev Endocrinol 11: 535-546, 2015.

9. Sawicka-Gutaj N, Zybek-Kocik A, Kloska M, Czarnywojtek A, Sowiński J, Budny B, Woliński K, Ziemnicka K, Mańkowska-Wierzbicka D and Ruchała M: Determinants of visfatin/NAMPT serum concentration and its leukocyte expression in hyperthyroidism. Horm Metab Res 50: 653-660, 2018.

10. Sawicka-Gutaj N, Andrusiewicz M, Czarnywojtek A, Waligórska-Stachura J, Biczysko M, Skrobisz J, Sowiński J and Ruchała M: Changes of nicotinamide phosphoribosyltransferase expressions in thyroid glands of patients with different thyroid pathologies. Biomed Res Int 2018: 1316390, 2018.

11. Sawicka-Gutaj N, Budny B, Zybek-Kocik A, Sowiński J, Ziemnicka K, Waligórska-Stachura $\mathrm{J}$ and Ruchała $\mathrm{M}$ : Nicotinamide phosphoribosyltransferase leukocyte overexpression in Graves' opthalmopathy. Endocrine 53: 497-504, 2016.

12. Ozkaya M, Sahin M, Cakal E, Yuzbasioglu F, Sezer K, Kilinc M and Imrek SS: Visfatin plasma concentrations in patients with hyperthyroidism and hypothyroidism before and after control of thyroid function. J Endocrinol Invest 32: 435-439, 2009.

13. Han J, Zhang TO, Xiao WH, Chang CQ and Ai H: Up-regulation of visfatin expression in subjects with hyperthyroidism and hypothyroidism is partially relevant to a nonlinear regulation mechanism between visfatin and tri-iodothyronine with various concentrations. Chin Med J (Engl) 125: 874-881, 2012.

14. Li JB and Li YB: The relationship between visfatin and autoimmune thyroid disease. Labeled Immunoassays Clin Med 17: $355-358,2010$

15. Livak KJ and Schmittgen TD: Analysis of relative gene expression data using real-time quantitative PCR and the 2(-Delta Delta C(T)) method. Methods 25: 402-408, 2001.

16. Bi TQ and Che XM: Nampt/PBEF/visfatin and cancer. Cancer Biol Ther 10: 119-125, 2010.

17. Chung CP, Long AG, Solus JF, Rho YH, Oeser A, Raggi P and Stein CM: Adipocytokines in systemic lupus erythematosus: Relationship to inflammation, insulin resistance and coronary atherosclerosis. Lupus 18: 799-806, 2009.

18. Li Y, Ke J, Peng C, Wu F and Song Y: microRNA-300/NAMPT regulates inflammatory responses through activation of AMPK/mTOR signaling pathway in neonatal sepsis. Biomed Pharmacother 108: 271-279, 2018.
19. Kong YY, Li GQ, Zhang WJ, Hua X, Zhou CC, Xu TY, Li ZY, Wang P and Miao CY: Nicotinamide phosphoribosyltransferase aggravates inflammation and promotes atherosclerosis in ApoE knockout mice. Acta Pharmacol Sin 40: 1184-1192, 2019.

20. Neubauer K, Bednarz-Misa I, Walecka-Zacharska E, Wierzbicki J, Agrawal A, Gamian A and Krzystek-Korpacka M: Oversecretion and overexpression of nicotinamide phosphoribosyltransferase/pre-B colony-enhancing factor/visfatin in inflammatory bowel disease reflects the disease activity, severity of inflammatory response and hypoxia. Int J Mol Sci 20: pii: E166, 2019.

21. Dayan CM and Daniels GH: Chronic autoimmune thyroiditis. N Engl J Med 335: 99-107, 1996.

22. Grani G, Carbotta G, Nesca A, D'Alessandri M, Vitale M, Del Sordo M and Fumarola A: A comprehensive score to diagnose Hashimoto's thyroiditis: A proposal. Endocrine 49: 361-365, 2015.

23. Ehlers M, Thiel A, Bernecker C, Porwol D, Papewalis C, Willenberg HS, Schinner S, Hautzel H, Scherbaum WA and Schott M: Evidence of a combined cytotoxic thyroglobulin and thyroperoxidase epitope-specific cellular immunity in Hashimoto's thyroiditis. J Clin Endocrinol Metab 97: 1347-1354, 2012.

24. Medzhitov R, Preston-Hurlburt P and Janeway CA Jr: A human homologue of the Drosophila Toll protein signals activation of adaptive immunity. Nature 388: 394-397, 1997.

25. Arbour NC, Lorenz E, Schutte BC, Zabner J, Kline JN, Jones M, Frees K, Watt JL and Schwartz DA: TLR4 mutations are associated with endotoxin hyporesponsiveness in humans. Nat Genet 25: 187-191, 2000.

26. Tatematsu M, Yoshida R, Morioka Y, Ishii N, Funami K, Watanabe A, Saeki K, Seya T and Matsumoto M: Raftlin controls lipopolysaccharide-induced TLR4 internalization and TICAM-1 signaling in a cell type-specific manner. J Immunol 196: 3865-3876, 2016

27. Nogueira AV, Nokhbehsaim M, Eick S, Bourauel C, Jäger A, Jepsen S, Cirelli JA and Deschner J: Regulation of visfatin by microbial and biomechanical signals in PDL cells. Clin Oral Investig 18: 171-178, 2014

28. Camp SM, Ceco E, Evenoski CL, Danilov SM,Zhou T, Chiang ET, Moreno-Vinasco L, Mapes B, Zhao J, Gursoy G, et al: Unique Toll-like receptor 4 activation by NAMPT/PBEF induces NFKB signaling and inflammatory lung injury. Sci Rep 5: 13135, 2015.

29. Sun S, He M, VanPatten S and Al-Abed Y: Mechanistic insights into high mobility group box-1 (HMGb1)-induced Toll-like receptor 4 (TLR4) dimer formation. J Biomol Struct Dyn 37: 3721-3730, 2019.

30. Taylor KR, Trowbridge JM, Rudisill JA, Termeer CC, Simon JC and Gallo RL: Hyaluronan fragments stimulate endothelial recognition of injury through TLR4. J Biol Chem 279: 17079-17084, 2004.

31. Swaroop S, Sengupta N, Suryawanshi AR, Adlakha YK and Basu A: HSP60 plays a regulatory role in IL-1 $\beta$-induced microglial inflammation via TLR4-p38 MAPK axis. J Neuroinflammation 13: 27,2016

32. Smiley ST, King JA and Hancock WW: Fibrinogen stimulates macrophage chemokine secretion through Toll-like receptor 4 . J Immunol 167: 2887-2894, 2001.

33. Alba R, Bosch A and Chillon M: Gutless adenovirus: Last-generation adenovirus for gene therapy. Gene Ther 12 (Suppl 1): S18-S27, 2005.

34. Bruzzone S, Fruscione F, Morando S, Ferrando T, Poggi A, Garuti A, D'Urso A, Selmo M, Benvenuto F, Cea M, et al: Catastrophic $\mathrm{NAD}^{+}$depletion in activated $\mathrm{T}$ lymphocytes through Nampt inhibition reduces demyelination and disability in EAE. PLoS One 4: e7897, 2009.

This work is licensed under a Creative Commons Attribution-NonCommercial-NoDerivatives 4.0 International (CC BY-NC-ND 4.0) License. 